\title{
La complejidad como base metodológica para la construcción de conocimientos en el Observatorio del Agua para el Estado de Veracruz, OABCC (Agua, Bosques, Cuencas y Costas)
}

\section{Complexity as a methodological basis for the construction of knowledge in the Water Observatory for the State of Veracruz, OABCC (Water, Forests, Basins and Coasts)}

\section{Socorro Menchaca Dávila ${ }^{a}$}

a Responsable del Observatorio del Agua para el Estado de Veracruz, Centro de Ciencias de la Tierra, Universidad Veracruzana, Xalapa, México.

Contacto: jmartinezb@uv.mx

Recibido: 09 de septiembre de 2020

Aceptado: 15 de octubre de 2020

RESUMEN: Se presenta, en términos generales, el marco epistémico del diseño y creación de las metodologías del Observatorio del Agua, que es la base para investigar objetos de estudio entendidos como sistemas complejos mediante enfoques interdisciplinarios; también se presentan las metodologías que se han construido en el marco de múltiples temáticas de agua, como medio para generar conocimientos.

Palabras clave: Metodología; sistema; complejidad; interdisciplina; agua.

ABSTRACT: The epistemic framework of the design and creation of the Water Observatory methodologies is presented in general terms, which is the basis for investigating objects of study understood as complex systems through interdisciplinary approaches; The methodologies that have been built within the framework of multiple water issues are also presented, as a means to generate knowledge.

Keywords: Methodology; System; Complexity; interdiscipline; water. 


\section{Introducción}

L

os fenómenos y problemáticas, que tienen como contexto la complejidad debido a que están determinados por la relación del hombre y/o la sociedad con el medio ambiente, son los que invariablemente se abordan en las múltiples temáticas del agua, que implican la interrelación e interdependencia de los ámbitos del medio ambiente, social y económico, y por ello, al desarrollo del enfoque interdisciplinario, trabajo académico que se desarrolla de manera permanente, tanto en el marco teórico/conceptual como en el metodológico del Observatorio del Agua para el estado de Veracruz (Agua, Bosques, Cuenas y Costas) para la generación de conocimientos.

Se señala por principio que el estudio y/o investigación de objetos de estudio complejos remite, por principio, a un cambio en el desarrollo del quehacer científico, el cual, desde hace siglos, ha sido primordialmente disciplinario, fragmentado, hiperespecializado; además, muestra la urgencia y/o necesidad de buscar e identificar lo que está unificado, interconectado, interrelacionado, que se traduce en la acción metodológica de crear nuevas aproximaciones de comprender los fenómenos; establecer nuevos esquemas epistémicos en el abordaje de las problemáticas; así como diseñar, desarrollar y proponer posibles respuestas útiles para la solución de los problemas que se abordan en su contexto de aplicación.

Se señala aquí que la investigación de objetos de estudio complejos en el contexto del ciclo natural y antrópico del agua, y las múltiples o diversas temáticas tiene una indiscutible relevancia debido a la alta pertinencia sobre el cambio en los modos de producción del conocimiento, ya que bajo el enfoque de la complejidad y el desarrollo de metodologías interdisciplinarias se logra una mayor comprensión de la problemática, cuyo aspecto clave es comprender los efectos que tiene el desarrollo de las actividades humanas en relación con los ecosistemas, y los aspectos físicos, químicos y biológicos.

En este artículo se expone el marco epistemológico general relacionado con la construcción de las metodologías que se generan en el OABCC, estableciendo una síntesis sobre la historia de la ciencia en relación con la simplificación de los fenómenos y la comprensión de la complejidad; la importancia del desarrollo de enfoques interdisciplinarios para la investigación de sistemas complejos; y también se enuncian las metodologías que se han diseñado y aplicado en el contexto del OABCC. 


\section{La complejidad como eje de la construcción de conocimientos socialmente pertinentes}

Según los registros sobre la evolución de la ciencia, ésta ha tenido como huella la simplificación respecto al estudio, análisis, explicación e interpretación de los fenómenos. Descartes, en su obra el Discurso del Método, inaugura formalmente la configuración de la ciencia, en donde se pondera lo matemático como presupuesto básico de lo científico bajo los preceptos de lo observable, medible, cuantificable y predecible, principalmente. Esto ha tenido como consecuencia que se privilegie significativamente el desarrollo de los atributos enmarcados dentro de un enfoque reduccionista y cuantitativo en el estudio de los diversos fenómenos, mismos que, además, pueden integrar diversos campos de conocimiento de las ciencias naturales y sociales.

A partir de lo anterior, se puede establecer que, en la historia de la ciencia, desde una perspectiva occidental, se ha instaurado una batalla constante contra la complejidad de los fenómenos, lo que ha signado al progreso de la ciencia, mediante el establecimiento de enfoques que ponderan la reducción de los ámbitos complejos a los elementos simples. Así, Ilya Prigogine (1994) establece que estamos todavía en la prehistoria de la ciencia: "Creo que estamos al comienzo de la ciencia, sólo comenzamos a ver la complejidad del mundo. La ilusión que antes teníamos provenía de la elección del objeto que habíamos hecho (por ejemplo, el péndulo), y luego extrapolábamos esos objetos simples para referirnos al mundo como totalidad. Esto ha causado confusión durante mucho tiempo, porque muchas de las cosas que hemos descubierto ahora podrían haber sido descubiertas mucho antes." De tal forma que, actualmente, se reconoce que la realidad representa a la complejidad misma, ésta es el ejemplo máximo de complejidad.

Un avance significativo en el progreso de la ciencia con relación a la comprensión e investigación de lo complejo, se centra en la termodinámica moderna donde se postula el noequilibrio en los sistemas, se reconoce que existen procesos de carácter irreversible y la heterogeneidad de sus componentes. Se señala, además, que la termodinámica ha sido considerada como el alma mater de los sistemas complejos que se define aquí como un conjunto de componentes y elementos heterogéneos que están relacionados con una red causal, de tal manera que cada componente está interrelacionado y es interdependiente con otros, en un lapso de tiempo dado (Menchaca, 2003). La heterogeneidad aquí alude a los componentes o áreas de conocimiento -medio ambiente, social y económico- y a los elementos que los integran, los que representan las disciplinas o saberes que se incluyen al 


\section{Observatorios - Observatorio del Agua para el Estado de Veracruz}

sistema complejo, el cual es pragmáticamente entendido como un objeto de estudio en el ámbito científico.

Edgar Morin (1982), respecto a lo establecido, afirma que todos los sistemas son una unidad compleja que integra distintos componentes y elementos, los que incluso pueden estar en antagonismo; además, afirma el autor que lo complejo está entrelazado conjuntamente, lo que constituye un tejido unido estrechamente, aunque sus partes sean extremadamente diversas, la complejidad es sin duda una diversidad organizada.

En adición a lo planteado, se señala que un sistema complejo es un recorte de la realidad que se establece como objeto de estudio; ejemplificando lo anterior, si ubicamos las distintas problemáticas en el contexto del ciclo natural y antrópico del agua, el sistema complejo implica que su estructura integra, de modo estricto, los ámbitos de lo ambiental, social, económico, cuyo funcionamiento está determinado por la interrelación e interdependencia de dichos ámbitos o componentes del sistema, lo que significa que cualquier cambio en uno de los componentes tendría afectaciones positivas o negativas en los otros que lo integran debido al dinamismo del sistema complejo, así, por ejemplo, si en el ciclo natural la disponibilidad del agua en cantidad y calidad varía debido a distintos factores, entonces esto afectará a los contextos sociales y económicos.

De acuerdo con lo planteado se afirma que la cosmología moderna nos muestra un camino que privilegia la investigación de sistemas complejos, el cual se basa en la conjunción de los fenómenos sociales y naturales, los que abarcan una gran diversidad de disciplinas, que pueden articularse mediante dispositivos metodológicos que permiten que el conocimiento se integre para que se genere un "plus" interdisciplinario, lo que representa una nueva cualidad y permite la comprensión holística de los fenómenos y de la problemática inmersa en sus diversos ámbitos y facetas de su funcionamiento.

Es importante establecer también que la interdisciplina es el enfoque que permite la investigación de sistemas complejos, ya que se dirige a "resolver" los vacíos que son resultado de la unidisciplinariedad cuando se abordan problemáticas complejas, en donde los fenómenos de lo natural, social y económico están presentes.

En adición a lo anterior, se señala que la producción de conocimientos disciplinarios, sólo representa un fragmento o segmento de un sistema complejo, según lo establecido por Rolando García (1994); mientras que la interdisciplina se establece aquí como un proceso que implica la acción de unir, articular, integrar y/o relacionar el conocimiento, es decir, establece la interacción de ciertas disciplinas con otras. Lo interdisciplinario es el punto de intersticio donde varias disciplinas están involucradas alrededor de un objeto de estudio, establecido 
como un sistema complejo. Así, lo interdisciplinario aparece como un "plus", entendido como un valor agregado que se genera a partir de una acción "entre" disciplinas y "entre sujetos" que se reúnen para lograr un fin determinado, encausado a generar conocimientos en un contexto de aplicación, e implica también la evaluación del conocimiento que se transfiere, por ello, existe una responsabilidad social respecto a la utilidad del conocimiento. Con esto se propicia una comprensión y respuestas de mayor alcance sobre el comportamiento de los fenómenos que implican los desafíos inmersos en la relación del individuo y/o sociedad con el medio ambiente y la problemática que en esta interacción subyace.

Con base en lo anterior, podemos afirmar que la interdisciplina es una metodología para producir un nuevo conocimiento y una aproximación pragmática para resolver problemas complejos. Puede decirse que lo producido a partir de un "entre" las disciplinas, el espacio y/o intersticio, creado a partir de la interacción de ciertas disciplinas con otras, es lo que posibilita la integración y/o innovación del conocimiento. La interdisciplina aparece como la modalidad metodológica que permite la creación de conocimientos socialmente útiles, pertinentes, relacionados con las demandas que establece una realidad compleja que es dinámica e impermanente, por naturaleza.

\section{Metodologías que se han construido y se desarrollan bajo el enfoque de complejidad en el Observatorio del Agua para el estado de Veracruz, OABCC (Agua, Bosques, Cuencas y Costas)}

El OABCC estudia los fenómenos y problemáticas complejas en el ámbito del ciclo natural y antrópico del agua, lo que se relaciona con las múltiples demandas de la sociedad, que deben ser atendidas por los gobiernos en sus distintos niveles y las instituciones de educación superior, lo que alberga una alta pertinencia y responsabilidad social. Las grandes áreas temáticas en términos generales son las siguientes: las afectaciones antrópicas al funcionamiento de los ecosistemas y sus servicios ambientales; la contaminación de ríos y cuerpos de agua; la medición de la disponibilidad del agua en cantidad, calidad y aprovechamiento del recurso respecto a los distintos usuarios; el cambio de uso de suelo; el alto riesgo y vulnerabilidad social en el contexto de daños por inundaciones y deslaves provocados por fenómenos hidroclimatológicos; la baja eficiencia en el aprovechamiento del agua; así como el análisis del marco regulatorio del agua, entre otros.

Se señala que todas las metodologías que se han diseñado en el OABBC han sido configuradas bajo el enfoque de los sistemas complejos y la modalidad de construcción de conocimientos 
interdisciplinarios, descritos anteriormente, que abarcan múltiples variables e indicadores que se integran las bases de datos del OABCC. Dichas metodologías se enuncian a continuación:

- Gestión y manejo integral de los recursos hídricos en cuencas.

- Impactos y afectaciones a los servicios ambientales ecosistémicos de bosques y cuencas hidrológicas por el efecto antrópico de los usuarios del agua, agrícola, pecuario, acuacultura, doméstico e industrial.

- Determinación de la relación entre la calidad del agua y factor antrópico por los usuarios del agua.

- Identificación de los impactos y afectaciones de los usuarios del agua en la contaminación por cadmio, detergentes, fenoles y micribiológicos.

- Variación diacrónica de la contaminación del agua, precipitación y cambio de usos del suelo.

- Disponibilidad de agua, gestión y manejo del recurso hídrico municipal y su uso racional y/o cultura del agua a escala local.

- Percepción y opinión sobre la eficiencia de las políticas públicas sobre disponibilidad de agua en cantidad, calidad, valor económico, pago por servicios ambientales y cultura del agua.

- Índice de escasez del agua.

- Tratamiento de aguas residuales domésticas por métodos naturales en el contexto de zonas rurales.

- Riesgo por degradación ambiental de cuerpos de agua e inundaciones.

Cabe señalar que la labor académica del OABCC relativa a la creación, innovación y desarrollo de metodologías es uno de los ámbitos más importantes, ya que se considera que la medición de variables e indicadores es fundamental para la observación del comportamiento de los fenómenos y las problemáticas del agua, desde la perspectiva de la complejidad; y así, desarrollar un proceso que permita formular nuevas peguntas y elaborar nuevas respuestas a problemas que han prevalecido durante muchos años en nuestra región y país. Al respecto, actualmente en el OABCC se trabaja en los ámbitos de riesgo en volcanes y resiliencia de los sistemas ecohidrológico, entre otros. 


\section{Referencias}

Morin, E. (1983). La complejidad viviente en El Método. La vida de la vida, Capítulo II. Madrid: Cátedra, 418 p.

Menchaca, S. (2003). Os Conhecimentos Social e Ambientalmente Úteis. En L. Aragón, G. Clüsenner (Eds.), Problemática do uso local e global da agua da amazonia. Brasil: UNESCO, UFPA, NAEA. 484-489 pp.

García, R. (1994) Interdisciplinariedad y Sistemas Complejos. En E. Leff compil. Ciencias Sociales y Formación Ambiental. España: Gedisa. 85-124 pp. 